\title{
MATHEMATICAL EXPLANATION AS PART OF AN (IM)PERFECT SCIENTIFIC EXPLANATION AN ANALYSIS OF TWO EXAMPLES
}

\begin{abstract}
Alan Baker argues that mathematical objects play an indispensable explanatory role in science. There are several examples cited in the literature as solid candidates for such a role. We discuss two such examples and show that they are very different in their strength and (im)perfection, although both are recognized by the scientific community as examples of the best scientific explanations of particular phenomena. More specifically, it will be shown that the explanation of the cicada case has serious shortcomings compared with the explanation of the case of Königsberg's bridges. We will argue that the latter is a perfectly reliable scientific explanation that employs mathematical reasoning whereas the former is not.
\end{abstract}

Keywords: philosophy of mathematics, Enhanced Indispensability Argument, mathematical explanation, Königsberg bridge problem, cicada case

Platonists in the philosophy of mathematics often use explicit arguments to defend their views. The Enhanced Indispensability Argument (EIA) formulated by Alan Baker is one of them. The argument takes the following form (Baker 2009: 613): ${ }^{1}$

(1) We ought rationally to believe in the existence of any entity that plays an indispensable explanatory role in our best scientific theories.

* Faculty of Philosophy, University of Montenegro, Danila Bojovića bb, 81400 Nikšić, Montenegro, e-mail: vladimirdr@ucg.ac.me, ORCID: https://orcid.org/oooo-ooo1-90461596.

${ }^{1}$ The EIA is a newer version of the so-called Quine-Putnam Indispensability Argument (IA). For more on IA, cf. Liggins 2008. 
(2) Mathematical objects play an indispensable explanatory role in science.

(3) Hence, we ought rationally to believe in the existence of mathematical objects.

In the second premise, it is claimed that mathematical objects play an indispensable explanatory role in science. There are several characteristic examples in the literature cited in support of this claim. Such are, for instance, the cicada case, the honeycomb, the bridges of Königsberg, and the asteroid belt around Jupiter. ${ }^{2}$ We will discuss two of these examples and show that they are very different in their strength and (im)perfection, although both are recognized by the scientific community as examples of the best scientific explanations of particular empirical phenomena. More specifically, we will show that the explanation in the cicada case has serious shortcomings compared with the explanation in the case of Königsberg's bridges. We will provide arguments showing, it seems to us, that the latter is, but the former certainly is not, an example of a perfect scientific explanation in which a mathematical explanation was used. We will prove that, in a methodological sense, the mathematical explanation in these examples is used in many different ways. In the first case, the mathematical explanation involved a complete mathematization of the problem, while in the second case the mathematical tool was used only as an auxiliary means in the entire explanation. It will be shown that these differences will directly determine the reliability of each of the explanations and, thus, their real role in supporting the platonic project.

\section{THE MATHEMATIZATION \\ OF THE KÖNIGSBERG BRIDGE PROBLEM}

Let us briefly recall the problem of the Königsberg bridges (KBP) and its most famous solution. The KBP is a practical task from the physical world. We can denote this practical task by $T_{F}$. Its solution was proposed by Leonhard Euler in the middle of the $18^{\text {th }}$ century. A part of the old Prussian city of Königsberg looked as shown in Picture 1:

\footnotetext{
${ }^{2}$ Cf., e.g., Pincock 2012: 205-206, Tallant 2013, Baker 2016, Barrantes 2019, Bianchi
} 2016, Colyvan 2018. 


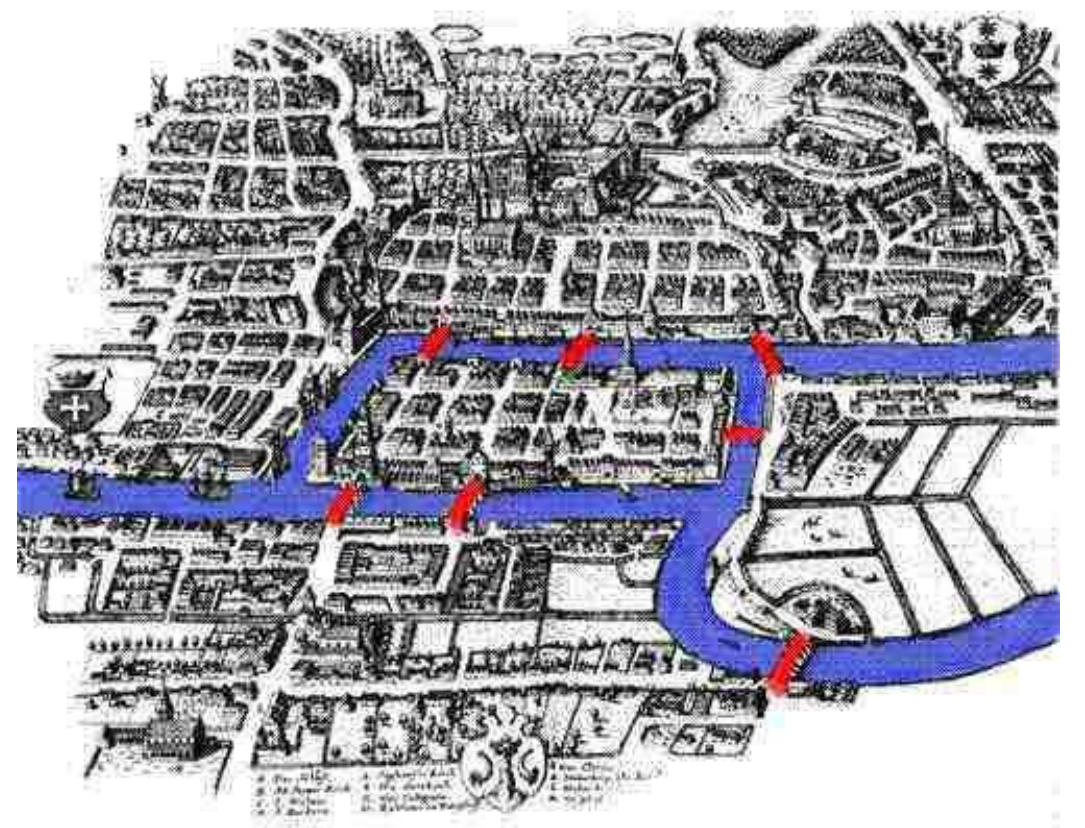

Picture 1. Bridges of Königsberg. Source: https://bit.ly/2repgKz

The Königsberg bridge problem asks whether the seven bridges of the city of Königsberg over the river Pregel can all be traversed in a single trip without doubling back, with the additional requirement that the trip ends in the same place it began. Euler's solution to the problem is based on the use of in terms of contemporary mathematics - graph theory. Namely, the entirety of the real situation can be represented by an abstract mathematical structure - a concrete graph. 3 To create a graph, several general steps must be taken. These steps ignore those objects in the picture that are irrelevant to solving

\footnotetext{
${ }^{3}$ Since the abovementioned statement may sound somewhat confusing or, even worse, logically incorrect, we will give a brief note here. When we talk about certain concepts and the abstraction that refers to them, then, especially in the sphere of mathematical theory, it is possible to speak of more levels of abstraction. Thus, for example, the concept $a$ can be the abstraction of the concepts $b, c, d$, etc., and the concept $a$ itself, thereby, represents the concretization of an even more general concept. In our case, the graph we are talking about will result from the generalization of the physical objects of a city. Of course, such generalization may include some other physical (either natural or artificial) configurations of physical objects. On the other hand, the graph we are about to speak of, with four vertices and seven branches, can be considered as a concretization of more general terms such as a graph which has four vertices, a graph that has seven branches, or simply a concept of a graph, the basic concept of graph theory.
} 
the problem, while those that are important have been preserved by being represented by symbols that are basic concepts of graph theory. Namely, objects that are located on the land, such as buildings, streets, parks, etc., are not considered. Then, each land entity bounded by the river is represented as a point, and each bridge that connects two such entities is a line that connects two corresponding points. Finally, by neglecting the river and the floating objects on it, graph $G$ is created (see Picture 2). 4

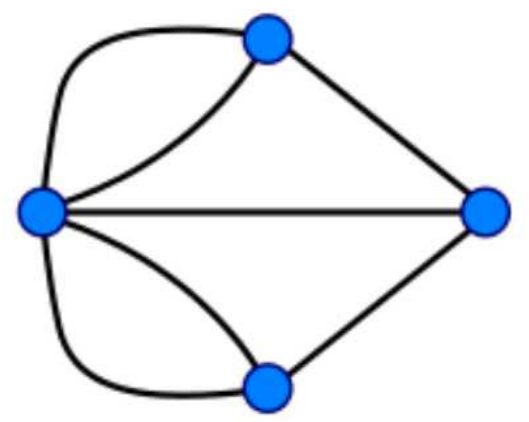

Picture 2. Graph $G$ : Euler's graph of Königsberg. Source: https://bit.ly/2sAQJXc

By this generalization, a practical problem from the physical world is reduced to a theoretical, mathematical question about graph $G$. Thus, the practical problem of crossing bridges formulated earlier can be re-formulated as a new mathematical task. We can denote it by $T_{M}$ : is it possible to traverse all the edges of graph $G$ once and only once, ending the walk in the vertex where it began? We can generalize the question even further by asking when it is possible to find such a path for an arbitrary graph - that is, to find Euler's tour. On the basis of a theorem of graph theory known as Euler's theorem, we find out that a necessary and sufficient condition for the existence of Euler's tour in a connected graph is the requirement that all vertices of the graph have even degree. ${ }^{5}$ Since all four vertices in graph $G$ have odd degree, it fol-

${ }^{4}$ In general terms, an arbitrary graph $G$ can be defined as an ordered pair $G=(V, E)$, with $V$ being a set of vertices, and $E$ being a set of edges that connect vertices. Since each edge connects two vertices of the graph, the elements of the set $E$ can be represented as two-membered subsets of the set $V$. For example, if the edge $\mathrm{e}_{k}$ connects vertices $\mathrm{v}_{i}$ and $\mathrm{v}_{j}$ then we can write $\mathrm{e}_{k}=\left\{\mathrm{v}_{i}, \mathrm{v}_{j}\right\}$.

5 Cf. Diestel 2005: 21-22. Having no desire to overload the text with mathematical details, we will define only a few basic terms in order to make the presentation clearer. If it is the same whether we are talking about edge $A B$ or edge $B A$, whereby this applies to all edges of the graph, we will say that the graph is undirected. A connected graph is such an undirected graph in which each pair of vertices can be joined by a path. The degree of a vertex is a natural number that shows how many edges come out of the vertex. 
lows that there is no Euler's path in it; that is, the Königsberg bridges cannot be traversed in a way described in $T_{F}$.

Let us now consider the extent to which the explanation/solution of the previous problem is convincing and justified and ask whether we could accept it without any doubt. We want to show that it is not possible to find a deficiency in this explanation of the problem from the physical world derived from mathematical means. When we say this, we do not refer, as it often happens in the context of explaining different problems, to the methodological aspects of the explanation, such as efficiency, elegance, inability to be simplified, or fertility. We have the veridic aspect of the explanation in mind. In other words, we believe that the truth of the statement to which the above explanation ultimately leads cannot be called into question. Let us try to make a case for this attitude.

First of all, we must point to an obstacle that will render our discussion unclear if we do not pay attention to it. At first blush, it may seem that the truth of a scientific explanation that essentially invokes mathematical considerations is beyond any doubt. Such a position can be justified by the respectable role that mathematics, being a role model and a deductive discipline, has among the sciences, but also based on a layman's belief in the indubitable truth of mathematical statements.

It is not easy to defend this position for at least three reasons. First, the question of the truth of the statements in mathematical theories is far more complicated than statements such as " $2+2$ always equals 4 ," or "all true statements in mathematics are provable" would suggest. The fact is that the concept of truth in mathematical theories relates, above all, to the question of the formal foundation of the statement, but modern mathematics raises many questions about the usability and justification of its own statements. ${ }^{6}$ Second, the use of mathematical explanations as a means of solving a scientific problem can be carried out in different ways. For example, some empiri-

\footnotetext{
${ }^{6}$ In this place, we do not want to address such issues, but we want to point to their existence and to the fact that neither mathematical nor philosophical community has spoken of them as the final word. For example, the field of axiomatization of basic mathematical theories, such as set theory, on the basis of which all other mathematical theories were constructed, abounds with questions. How to choose a concrete set of axioms? What is the justification for the chosen set of axioms? Does the chosen set of axioms ensure the construction of a consistent theory? How to justify statements such as the continuum hypothesis and the axiom of choice? These are just some of the questions that give rise to a great deal of debates in mathematical and philosophical circles. Cf., e.g., Maddy 1990. Also, Gödel's incompleteness theorems have further demonstrated the complexity of the question of the foundation of mathematical theories and the problems related to the existence of constraints of formal systems. Cf., e.g., Smullyan 1992.
} 
cal explanations can be fully mathematized, while in other cases, the empirical phenomenon can be explained by a mathematical tool only partially that is, in combination with some other non-mathematical aids.7 It follows that the power of mathematical arguments is not used to the same extent in the explanation of all phenomena, if it is used at all. Finally, when we speak of a mathematical explanation of empirical phenomenon $P$, we should bear in mind that our goal is not to consider the truth of the mathematical statements but the truth of the statements relating to $P$, which is, to some extent, explained by mathematical statements. It is clear that the truth of statements of one type is to some extent related to the truth of the other kind of statements, depending on how and in what measure a mathematical tool is used in explaining the scientific phenomenon. However, this does not mean that the truth of a statement of one type implies the truth of all statements of another type. Namely, it is possible to use mathematical facts in a concrete explanation of the empirical phenomenon, but that explanation, generally speaking, is unreliable; that is, it leads to a problematic result/statement. ${ }^{8}$

Let us go back to the KBP and its explanation. Moving the consideration of the problem from Picture 1 to Picture 2 is an example of reducing the empirical situation to an abstract model presented by a mathematical object. The second picture ignores all objects from the first picture that are not relevant to the solution of the problem. Thus, if we were to observe only the second picture without knowing how it originated, it would be difficult to get the idea that it was a kind of map of a certain city. There are no houses, streets, or other city facilities in it. There is no river and no floating objects on it. On the other hand, the objects relevant to the solution of the problem are retained but abstracted. Thus, the symbols of the land entities are there, but they have

\footnotetext{
7 When we say that the explanation of a scientific phenomenon is completely mathematized, we do not want to say that this explanation is absolutely independent of any nonmathematical (physical, chemical, etc.) claims. Namely, any explanation of a scientific phenomenon presupposes perception (either directly by senses or indirectly by technical means, such as microscopes, telescopes, simulators, etc.) of specific physical details related to the phenomenon. In this context, any explanation is certain depending on certain empirical claims. Whether this perception is reliable or not, is not the subject of this paper. However, once the empirical phenomenon has been perceived, its explanation can take different methodological paths. One of them is purely mathematical as in the KBP. In this case, we are talking about a complete mathematization of the explanation. On the other hand, there are explanations that include non-mathematical claims that go beyond the initial perception of the problem. An example of this explanation will be analyzed in the next section.

${ }^{8}$ An example of such a statement is the result of an explanation of a natural phenomenon that will be described in the next section.
} 
completely changed shape in relation to the original objects they represent. They have become points. Although the rivers and their symbols are no longer represented in Picture 2, the bridges connecting land entities are represented by lines. Of course, the idea of symbolizing a concrete physical situation from Königsberg could have been made in infinitely many different ways. The land entities and bridges between them and other physical objects from Picture 1 could be represented by some other geometric objects. However, the symbolization in Picture 2 provides sufficient conditions for a mathematical solution of problems from the physical world. On what basis can we claim this?

First, the physical structure of the city is selectively mapped onto a mathematical structure. The problem solution has moved from the field of the physical world to the field of mathematical theory. Second, since only objects from the physical world that are relevant for solving the Problem the land entities and bridges between them, in the configuration and interrelationships they have in the physical world - are mapped in the mathematical structure, every statement that is provable and refers to graph $G$ has its true counterpart in the city of Königsberg. In other words, it cannot be the case that some statement regarding graph $G$ is true (or not true) while the corresponding counterpart of this statement relating to the situation in Königsberg has the opposite truth value (not true or true, respectively). Of course, the degree of complexity of the statement that we can see in the graph is irrelevant. For example, it is not a true statement that in graph $G$ there is no vertex starting from which, using up to three edges, we can visit all the remaining vertices. The case is the same with the counterpart of this statement: in Königsberg, there is no land entity starting from which we can go to all the other land entities using up to three bridges. The situation does not change even when it comes to more complex statements such as that expressed by Euler's theorem. Since it has been proven and, therefore, since it is a true mathematical statement that it is not possible to find Euler's path in graph $G$, it follows that the statement about the impossibility of finding the path in Königsberg as formulated in task $T_{F}$ is true.

The previous consideration, however, is not a proof of the methodological statement that the solution of the mathematical problem $T_{M}$ leads to a true statement relating to task $T_{F}$. We want to show with more formal details not only that there is a direct veridical connection between the solutions of tasks $T_{M}$ and $T_{F}$, but also that for every true statement relating to graph $G$ we can find the appropriate true statement about the objects in Königsberg, and vice versa. How do we justify this statement? 
As we have just said, the statement has, above all, a methodological character, as it relates to different practical and theoretical approaches to solving a problem. However, its justification can be mathematical again, but now only in a meta-sense. 9 First of all, as we have mentioned earlier, it is obvious that graph $G$ presents all the objects that are relevant to solving a specific problem. In our case, these are bridges and land entities. Second, we have noted that such symbolization - that is, representation of physical objects by points and lines - although quite informal, is not only a relation between two sets of objects but also a mapping between them. Let us describe this mapping more precisely. Its domain, denoted by $A$, is a subset of a set of Königsberg's physical objects. This subset is made up of physical objects relevant to solving task $T_{F}-$ bridges and the land entities. The codomain of the mapping - we can denote it by $B$ - consists of elements of graph $G$ - that is, its vertices and edges. Let us denote this mapping by $f .{ }^{10}$

We will note, in addition, that $f$ is a bijection. Generally speaking, the fact that a mapping is a bijection between two sets allows us to identify in a certain way those sets in the context of their cardinality, thus abstracting from the kind and nature of the objects of both sets. However, this fact is insufficient to provide a direct connection of truth between the statements relating to tasks $T_{F}$ and $T_{M}$. Indeed, from the fact that $f$ is a bijection, we cannot infer the information whether this mapping onto a codomain maps not only the elements of domains but also the relationships that exist between them. In our two tasks, both physical and mathematical, there are two relations, one between the domain elements and the other between the codomain elements. Regarding these relations, we note that mapping $f$ is slightly more than a simple bijection. Namely, it is a kind of isomorphism, a bijection that "preserves" the structure/relation that exists in the domain of this mapping. ${ }^{11}$

\footnotetext{
9 When we use the term "meta," we mean that the mathematical apparatus will be used to justify the claim about a direct truthful connection between mathematical and physical statements.

${ }^{10}$ Binary relation $f \subseteq A \times B$, where $A$ and $B$ are nonempty sets, is a mapping from $A$ to $B$ if $(\forall x \in A)(\exists ! y \in B) y=f(x)$. Cf., e.g., Düntsch, Gediga 2000: 35. The relation in our case, that of symbolizing the objects of set $A$ by the objects of set $B$, satisfies the previous condition. Indeed, exactly one object of set $B$, an edge or a vertex, is assigned to each object of set $A$, a bridge or a land entity, respectively.

${ }^{11}$ Since the concept of relation is used in different contexts in this place, additional clarification is needed. Here we have talked about the binary relation $f$ of "symbolizing" between sets $A$ and $B$, which turns out to be a function. Now, we are talking about two relations of which one is defined in the set $A$ and is denoted by $\rho_{F}$, and the other is defined in the set $B$ and is denoted by $\rho M$. If the set of land entities $L E=\left\{l e_{1}, l e_{2}, l e_{3}, l e_{4}\right\}$, and the set of bridges $B R=\left\{b_{1}, b_{2}, \ldots, b_{7}\right\}$, then it is the case that $\rho_{F} \subseteq L E \times B R \times L E$, where the ordered
} 
In other words, all relationships that exist between objects in the domain also exist in the analogous sense and between the corresponding objects of the codomain. In this way, by mapping $f$ from the domain to the codomain, the identical layout of all the objects relevant to the solution of our problem that is, the corresponding connections which existed between them in the domain - is mapped. Owing to these properties of mapping $f$ we can say that for each relationship that exists in graph $G$ there is a corresponding relation in Königsberg, and vice versa. It follows that it is not possible for any statement relating to the objects of graph $G$ to have one truth value while the Königsberg counterpart of that statement has another one. For any proven statement referring to graph $G$ there is a true statement that relates to the movement over bridges and land entities of Königsberg. Thus, the practical problem of task $T_{F}$ is completely mathematized and reduced to a theoretical discussion in task $T_{M}$. Every proven statement in graph $G$ ensures the existence and accuracy of the corresponding counterpart in Königsberg, and vice versa.

\section{EXPLANATION OF THE CICADA CASE: MATHEMATICS IN THE SERVICE OF SCIENTIFIC ASSUMPTIONS}

Another example used as an illustration of a mathematical explanation of empirical phenomena, and also marshalled in support of the platonic claim in the second premise of the Enhanced Indispensability Argument, is the wellknown cicada case (CC). ${ }^{12}$ Let us recall the basic details of this example:

The example featured the life cycle of the periodical cicada, an insect whose two North American subspecies spend 13 years and 17 years, respectively, underground in larval form before emerging briefly as adults. One question raised by biologists is: why are these life cycles prime? It turns out that a couple of explanations have been given that rely on certain number theoretic results to show that prime cycles minimize overlap

triplet $\left(l e_{i}, b_{j}, l e_{k}\right) \in \rho_{F}(i, k=1, \ldots, 4),(j=1, \ldots, 7)$ if and only if there is a bridge $b_{j}$ between the land entities $l e_{i}$ and $l e_{k}$. In a similar way, we can describe the relation $\rho_{M}$ as a subset of the Cartesian product $V \times E \times V$, where $V$ is a set of vertices, and $E$ is a set of edges of graph $G$. In this case, the triplet $\left(v_{i}, e_{j}, v_{k}\right) \in \rho_{M}(i, k=1, \ldots, 4),(j=1, \ldots, 7)$ if and only if in graph $G$ there is a connection $e_{j}$ between the vertices $v_{i}$ and $v_{k}$. When we say that $f$ is an isomorphism, a bijection that preserves a relation from the domain, we want to point out that the structure of the domain, the relation of its elements, is preserved in the structure of the codomain in relation to its elements. To say formally, if it is $\left(l e_{i}, b_{j}, l e_{k}\right) \in \rho_{F}$ then $\left(v_{i}, e_{j}, v_{k}\right)$ $\in \rho_{M}$, where is $f\left(l e_{i}\right)=v_{i}, f\left(b_{j}\right)=e_{j}$ and $f\left(l e_{k}\right)=v_{k}$.

${ }^{12}$ This example has already been presented in detail in many places. Cf., e.g., Baker 2005, Bangu 2008, Panza, Sereni 2013: 209, Lange 2013: 498-499. The example has a new version in Baker 2016, but we will deal with the original version given in Baker 2005. 
with other periodical organisms. Avoiding overlap is beneficial whether the other organisms are predators, or whether they are different subspecies. (Baker 2009: 614)

For example, a prey with a 12-year cycle will meet - every time it appears - properly synchronized predators appearing every $1,2,3,4,6$ or 12 years, whereas a mutant with a 13-year period has the advantage of being subject to fewer predators. (Goles, Schulz, Markus 2001: 33).

Thus, an explanation of one natural phenomenon is given - the fact that the lifespan of a particular animal is expressed exclusively by a prime number. Although the aforementioned phenomenon belongs primarily to biology, this question was raised primarily from a mathematical angle. Only prime numbers are found when it comes to the lifespan of the insect. The question itself contains a specific mathematical concept, and it would be expected that the explanation must be based on mathematical means and facts.

Before analyzing the explanation of the phenomenon, let us look at what reason could be an incentive to consider the explanation at all. Is there anything extraordinary in the fact that the life cycle of North American crickets is not a composite number in any of the two recorded cases? In other words, is it possible that this fact is a completely random event undetermined by any biological, mathematical, or some other fact? On the basis of the elementary concepts of probability theory, we can say that the answer to this last question is affirmative. However, it is not difficult to notice that the probability of that number's being prime is significantly lower than the likelihood of its being composite. ${ }^{13}$ Bearing in mind that in both cases of our phenomenon (in both subspecies) we have registered a prime number, which is a random event with even lower probability, it seems that we can speak of justified reasons for raising the issue regarding the $\mathrm{CC}$.

Let us now turn to the explanation of the CC. It is based on several facts, but also on some assumptions. The first fact mixes biology and mathematics: the registered lifespans of cicadas are expressed in numbers 13 and 17. The second fact is mathematical: a prime number is divisible only by two numbers - by 1 and by itself, while a composite number has more than 2 divisors. The third fact is biological: avoidance of predators facilitates the survival of a single animal and thus of the entire species. The first assumption is biological: the predators of cicadas, as well as cicadas, have life cycle length in which

\footnotetext{
${ }^{13}$ It is easy to see that the probability that a randomly selected natural number between, for example, numbers 10 and 20, including those two numbers, will be prime, is almost two times smaller than the probability for such a selected number to be composite. This closed interval of natural numbers has been chosen since it approximately covers the numerical values obtained in the CC. It is also easy to check that the smaller displacement of the interval limits will not significantly affect the value of the obtained probability.
} 
they periodically emerge. Finally, the second assumption relates to evolution: during evolution, organisms have developed certain characteristics that give them a better chance of survival. Thus, on the basis of previous statements, it is possible to find an explanation for the CC. The observed animal has evolved into a species whose lifespan is expressed by a prime number for very pragmatic reasons. Namely, if the life cycle length of cicadas is expressed by a prime number, assuming that the predators have some life cycles in which they appear, the number of predators that will meet the cicadas is reduced to a minimum. For example, when the insect life cycle length is 17 years, then it has the chance to meet predators whose life cycle lengths are 1 or 17 years because the number 17 is divisible only by 1 and 17. On the other hand, if, for example, the insect life cycle length were 18 years, then it would have a chance to encounter predators whose life cycle of appearances is $1,2,3,6$, 9, and 18 years.

Let us see to what extent the explanation of the $\mathrm{CC}$ is reliable and convincing. The fact that this is an example of a mathematical explanation of a scientific phenomenon might perhaps have prompted us to think that it is a "safe" solution that can never be doubted. However, on the basis established in the previous paragraph, it seems to us that there are several reasons why the explanation can be questioned. First, the mathematical tool and the mathematical statements used in the explanation represent only a part of all the tools and statements that have been used in it. Indeed, apart from the facts drawn from number theory that follow from the definition of a prime and composite number, biological and evolutionist statements were also used. Some of them cannot be unconditionally trusted, because there are competing scientific theories that do not support them. Thus, the explanation of the CC is not entirely mathematical. The truth of the non-mathematical part of the explanation is not guaranteed, which calls into question the reliability of the whole explanation. Finally, the initial problematization of the target phenomenon, the search for a relationship between the life cycle length and special types of numbers, is based on probability. The probability of an event that the life cycle is expressed by a prime number is significantly lower than the probability of the event that the life cycle will be expressed by a composite number. However, this fact does not mean that the first outcome is not achievable by chance; that is, it does not mean that it must be achieved owing to the influence of certain biological and evolutionist reasons related to encounters with predators. In other words, it is possible that thinking in terms of probability is likely to lead the investigation in the wrong direction.

Furthermore, we want to highlight the first of the two assumptions mentioned in the explanation because, in our opinion, its status is particularly 
"unsafe." More specifically, no proof has been given in support of its truth. It appears that because cicadas emerge in certain life cycles, it is assumed that such an appearance of cicadas should also have their predators. However, why should predators have such life cycles? In the explanation, we have no proof for such a statement, nor a single predator that lives in such a way. In order not to take this consideration as empty speculation, let us point out that mathematical biologists themselves reveal that there is still no predator of cicadas with similar periodical activities in nature (see Behncke 2000: 417, Lloyd, Dybas 1966: 146). Until we have at least one such example, what is most likely is that predators live an unspecified number of years without any life cycles. If so, if we do not have predators with life cycles, the whole explanation must be thrown away because it will be completely unimportant if cicadas live 17 or 18 years. The lifespan of cicadas expressed by a prime number will not be an advantage for survival in relation to the case when this life cycle length is expressed by a composite number. The number type will not be relevant for survival.

Finally, the third reason that may cast doubt on the explanation of the CC relates to one assumption that is not mentioned in the explanation, but it is implied. Let us consider, regardless of the argument from the previous paragraph, that there are predators with life cycles. In such a case, would the lifespan of a cicada expressed by a prime number be an advantage in its survival? Yes, it would, but with the additional requirement - the lifespan of a predator needs to be shorter than the lifespan of the cicadas. Let us explain this detail. If the lifespan of a predator (PL) were shorter than the lifespan of a cicada (CL), assuming that CL is a prime number, then the time between their two encounters would be maximized, giving the cicada greater chances of survival. Namely, that time would be defined as the least common multiple (LCM) of the PL and CL numbers. If there were PL $<\mathrm{CL}-$ that is, if PL and CL were coprime integers - then it would be the case that LCM(PL, CL) $=$ $\mathrm{PL} \times \mathrm{CL}$. Hence, the LCM of two numbers would be their product, having the highest value that LCM, theoretically, can have for two numbers. ${ }^{14}$ On the other hand, if we had PL > CL, then the fact that CL is a prime number would not be a sufficient condition for the time of re-encounters between cicadas and their predators, expressed by LCM(PL, CL), to be maximized. Namely, in general, there would be no LCM $(\mathrm{PL}, \mathrm{CL})=\mathrm{PL} \times \mathrm{CL}$. Indeed, if, for example, CL

${ }^{14}$ If, for instance, $a=17$ and $b=9$, then $\operatorname{LCM}(a, b)=17 \times 9$. In general, if the natural numbers $a$ and $b$ are coprime integers, then $\operatorname{LCM}(a, b)=a \times b$. It is clear that it can never happen that $\operatorname{LCM}(a, b)>a \times b$, because it is the least common multiple. Indeed, if we allow $\operatorname{LCM}(a, b)$ to exist such that $\operatorname{LCM}(a, b)>a \times b$, then it will not be the least common multiple, since $a \times b$ is also the common multiple of these two numbers. 
$=x$, where $x$ is a prime number, and PL $=2 x$, then LCM $(\mathrm{PL}, \mathrm{CL})=2 x \neq 2 x^{2}=$ $\mathrm{PL} \times \mathrm{CL} .{ }^{15}$ In other words, the property "is prime" referring to the number expressing the CL would not be an advantage for the survival of the species in the case when PL > CL, as it would be in the case of CP > PL. However, as we have already seen, we have neither biological arguments for the claim that the predators have life cycles nor, a fortiori, any arguments for the view that these life cycles are shorter than the cycles of cicadas. ${ }^{16}$

We have recalled the example of the $\mathrm{CC}$ and pointed to some of the reasons why it seems to us that its explanation can be questioned. We do not pretend to have exhausted the set of all possible remarks that can be made to the explanation of this example, but it seems to us that the three that we have set out represent sufficiently strong arguments to be reckoned with by anyone who would try to defend this explanation.

\section{TWO DIFFERENT ROLES \\ OF MATHEMATICAL EXPLANATION IN SCIENCE}

The two examples analyzed in the previous sections have at least one common thread - they are often listed in the literature as illustrating the application of a mathematical tool in explaining empirical phenomena. Indirectly, such examples attempt to support the second premise of the EIA that is, the claim that mathematical objects are indispensable for explaining scientific phenomena. We are not going to deal here with the question of whether the two examples illustrate the indispensability of the explanatory role of mathematics in science. ${ }^{17}$ However, what seems certain is that they illustrate the explanatory role of mathematical objects in science. In both ex-

${ }^{15}$ The consideration is similar if $\mathrm{PL}=n \times x$ or $\mathrm{PL}=x^{n}(n \in N)$.

${ }^{16}$ For a similar criticism, cf. Dieveney 2018.

${ }_{17}$ The question of the indispensability of mathematics to science has been raised through the so-called Quine-Putnam indispensability argument (IA). The fundamental difference between the IA and the EIA is that in the latter argument the emphasis is put on the indispensability of the explanatory role of the mathematical tool in science. Historically speaking, the EIA is an "improved" version of the IA, according to which the role that mathematical objects have in describing and explaining empirical phenomena is reduced to quantification and indexing of the physical objects. However, there are arguments that show that the notion of indispensability in the EIA, in connection with the explanatory role of mathematical objects in science, is not precisely defined and that it is not easy to determine the domain of the EIA - that is, the space of the mathematical objects to which the argument relates. Cf. Drekalović 2016, Drekalović, Žarnić 2018, Drekalović 2018. 
amples, mathematical tools are used to explain some empirical phenomenon. But a warning is in order. Over many centuries, mathematics has achieved a special, privileged position among scientific disciplines. With its deductively built theories, it is often taken as a model that the other sciences may try to emulate. Proof, as the main tool of mathematical justification, is a methodological ideal that many disciplines, formally or informally, are pursuing. The reason is understandable: a correct proof is an explicit and hardly disputed argument that justifies a mathematical claim. This feature of mathematical explanation, as well as the position that this procedure holds among other methodological tools used in the sciences, can lead us astray when it comes to the evaluation of scientific explanations. Namely, it may be tempting to think that the formal force of mathematical proof is transferred every scientific explanation that contains a mathematical explanation. The example we have analyzed in the third section shows that this is not the case.

We wanted to show that, on the one hand, there are examples of scientific explanations showing the full strength of a mathematical explanation. However, we also wanted to show that, on the other hand, there are examples of scientific explanations in which the authority of mathematical facts and mathematical methodology is, in a certain sense, "misused": we are talking here about examples in which mathematical facts or methodology are used for the purpose of scientific explanation, but these explanations are based, inter alia, on claims, assumptions, and hypotheses that make the entire explanation less than perfectly reliable. It seems to us that, on the basis of the analysis carried out in the previous two sections, it is possible to say that the Königsberg bridge problem (KBP) is an example of the first situation, while the cicada case (CC) is an example of the second type. The corresponding explanations of empirical phenomena that both involve a mathematical explanation differ in three important respects:

1) the manner and scope of the application of mathematical explanation in explaining the empirical phenomenon;

the role of the non-mathematical part of the explanation in the entire explanation; reliability of the entire explanation.

Considering the first aspect, we can say that in the KBP a phenomenon from the physical world is mapped by mathematical isomorphism onto a model that is exemplified by a concrete mathematical object - the graph. As we have shown, the graph can be viewed as a codomain of mapping whose domain is a set of all the objects in Königsberg that are relevant to the problem. As 
a mapping that preserves structure, isomorphism is a guarantee that all the considerations about the graph will have their counterparts in Königsberg. Thus, the whole problem situation is mapped from the physical world onto a mathematical structure. Let us recall that the mathematical tool is used on two levels. First, to reduce the physical phenomenon to mathematics, and then to solve a mathematical problem. Isomorphism has been used in the first part, while the theorem of graph theory has been used in the second part. In other words, a physical phenomenon is "safely" mapped onto a field of mathematical theory, where only objects relevant to the problem are represented. The rest of the explanation is entirely mathematical and could be considered without any connection with the physical world.

By contrast, the methodology of using mathematical tools in the CC is quite different. The problem has not been abstracted and mapped from the physical world onto the space of some mathematical theory, while differentiating between relevant and irrelevant objects. The problem remained in this world with an ad hoc one-time assistance of a mathematical tool. Facts about the number of divisors of prime and composite numbers are only auxiliary information that fits into the main explanatory thesis: evolution develops those biological properties of living beings that contribute to their survival. Since the scientific problem has not been reduced to a mathematical one, its solution cannot be mathematical either. The mathematical tool is there only to fill gaps in the reasoning guided by the evolutionary thesis. These gaps could not be filled without the basic facts of number theory. ${ }^{18}$ Therefore, this tool was a necessary tool; without it the whole explanation could not be given.

Let us also note a detail in which the KBP is "less" mathematical than the CC. Namely, the initial motivation for examining the KBP has nothing to do with mathematics, while in the $\mathrm{CC}$ this motivation is par excellence mathematical. In the former case, we are dealing with a practical question - a specific way of moving through space in the physical world, while in the latter we are considering a mathematical detail - the lifespan is expressed by a special type of natural numbers.

The second detail that we want to emphasize, and according to which the $\mathrm{KBP}$ and the $\mathrm{CC}$ can be distinguished, relates to parts of the explanations that are not mathematical ones. In the KBP, we can say that the non-mathematical part of the explanation appears only in the formulation of the initial problem and of its final solution. First, the question is raised about the possibility of a certain kind of movement in the physical world and then the entire problem

\footnotetext{
${ }^{18}$ Until this moment, in the literature dealing with this phenomenon, we have not found an evolutionary explanation of the phenomenon without using number theory, which, of course, does not exclude such a possibility in the future.
} 
and its solution are transferred to the field of mathematical theory. The mathematical model is first considered and then the solution to the problem is given through an explicit statement and taken back again to the physical world. The non-mathematical part of the explanation is static; that is, the non-mathematical objects to which the problem relates have not been used to provide the explanation. The relevant physical objects are mapped onto mathematical ones, and the essence of the explanation is derived in mathematical space, using mathematical tools only. There are no assumptions or facts from the physical world in it.

Things in the $\mathrm{CC}$ are quite different. The explanation is always located in the physical/biological world. The main assumptions that the explanation relies on are non-mathematical. The mathematical tool is used only to support the application of such assumptions in the concrete case. Therefore, the use of mathematical explanation in the entire explanation is conditioned, inter alia, by using the main assumptions in the explanation. ${ }^{19}$ Also, it would have been difficult to construct the explanation if it had not used assumptions that have no connection with mathematics - namely, the statement about the cyclic appearance of the predator and the statement that these cycles are shorter than the cycles in which cicadas appear.

At this point, a clarification should be made regarding the different strengths of the explanation of the KBP, on the one hand, and the explanation of the CC, on the other. These strengths are directly related to the reliance on non-mathematical assumptions in the explanation. Namely, someone might say that the explanation of the KBP is based on various non-mathematical assumptions, as well as the explanation of the $\mathrm{CC}$, and that, therefore, the former explanation is not much more reliable than the latter. Indeed, we could say that some preconditions have been made in the first explanation, for example, that the system of bridges is stable, that the "crossability" of the bridges is constant in time, or even that the notion of "crossing the bridge" makes sense, etc. Finally, appreciating the importance of extreme skepticism, we could say that we also assumed the existence of Königsberg. ${ }^{20}$ However, when we say that there are seven bridges in Königsberg, that they are given in the configuration as in Picture 1, that it is possible to cross over them, etc., we think about empirical claims that, at least in this paper, we do not try to challenge. In the same way, we do not try to challenge the empirical claims that the crickets appear every 13 or 17 years in the CC, that after birth they go underground, etc. Therefore, in our analysis, in both cases, we do not ques-

\footnotetext{
19 We say "inter alia" because the problem setting, in which mathematical concepts appear, predetermines the use of mathematical tools in the entire explanation.

${ }^{20}$ I thank an anonymous reader for having brought this important point to my attention.
} 
tion the registered/observed empirical claims on the basis of which the explanations are made. Of course, it is possible to challenge these claims, but it would go beyond the scope of our discussion.

On this basis, we can draw a conclusion regarding the reliability of the two explanations we have analyzed. The explanation of the KBP is an illustration of the type of explanation to which it is not easy to find a serious objection. ${ }^{21}$ It draws this power from the fact that it is completely mathematized - that is, formalized. It is not based on any assumptions, except on the assumption that a certain state of affairs has been observed at the beginning, which could call this explanation into question. By contrast, the explanation of the CC is based on such assumptions. Therefore, the whole explanation is uncertain and lies in the sphere of hypothesis and probability.

To summarize, although both cases we have analyzed illustrate the explanatory power of mathematics in science, we have attempted to show that they differ in important respects. On the one hand, the explanation of the KBP is a representative example showing how one empirical phenomenon can be described and explained, for the most part, mathematically, which ensures that the explanation is highly reliable. On the other hand, the explanation of the CC illustrates the possibility of such use of a mathematical tool in a scientific explanation that does not entail perfect reliability. ${ }^{22}$

${ }^{21}$ Let us note that the number of objects relevant to solving the KBP is rather "small," so the solution of the problem could possibly be made by random attempts and errors, without the use of mathematical tools. However, the significance of the explanation of the $\mathrm{KBP}$ is that the same methodology used in this explanation could have been implemented in the case when an arbitrary number of relevant physical objects were given. The mathematical process would essentially be the same regardless of the size of this number. Everything would be reduced to examining the number of links that vertices have in the graph. However, if the number of relevant physical objects was expressed by a relatively "large" natural number, the explanation would not be attainable by random attempts.

${ }^{22}$ On the basis of the examples analyzed in this paper, the reader might get the impression that the mathematical tool used to explain scientific phenomena is always trivial, and that such mathematical explanations can always be understood by a layman (I thank an anonymous reader who turned my attention to this detail). However, this is not true. The proof of this is the already mentioned example of honeycomb, which is more than twenty centuries old, and which was solved by a rather sophisticated mathematical tool twenty years ago. Namely, the problem is found already in the writings of Marcus Terentius Varro from the 1st century BC (Varro 1934), and it was solved in 1999 by a mathematician Thomas C. Hales (2001). The question is: why is the shape of the basic units of a honeycomb exactly the regular hexagon, and not some other regular or irregular polygon? The answer is based on a rather complex apparatus of the theory of optimality that a layman can hardly understand. Namely, it is shown that the optimal figure in the organization of the honeycomb, when it comes to the volume of the honeycomb unit and thus the consumption of materials for the construction of the bee habitat, is exactly the shape of a regular hexagon. 
Examples of the first type, by means of arguments such as the EIA, which use facts about the explanatory power of mathematics in science, can lend significant support to the platonic argument regarding the ontology of mathematical objects. On the other hand, examples of the second type, given their shortcomings, not only fail to provide analogous support but, on the contrary, can undermine the platonic position.

\section{BIBLIOGRAPHY}

Baker A. (2005), "Are There Genuine Mathematical Explanations of Physical Phenomena?," Mind 114(454), 223-238. https://doi.org/10.1093/mind/fzi223

Baker A. (2009), "Mathematical Explanation in Science," British Journal for the Philosophy of Science 6o(3), 611-633. https://doi.org/10.1093/bjps/axpo25

Baker A. (2016), "Parsimony and Inference to the Best Mathematical Explanation," Synthese 193(2), 333-350. https://doi.org/10.1007/s11229-015-0723-3

Bangu S. (2008), "Inference to the Best Explanation and Mathematical Realism," Synthese 160(1), 13-20. https://doi.org/10.1007/s11229-006-9070-8

Barrantes M. (2019), "Optimal Representations and the Enhanced Indispensability Argument," Synthese 196(1), 247-263. https://doi.org/10.1007/s11229-017-1470-4

Behncke H. (2000), "Periodical Cicadas," Journal of Mathematical Biology 40(5), 413-431. https://doi.org/10.1007/s002850000024

Bianchi S. (2016), "Which Explanatory Role for Mathematics in Scientific Models? Reply to 'The Explanatory Dispensability of Idealizations'," Synthese 193(2), 387-401. https:// doi.org/10.1007/s11229-015-0795-0

Colyvan M. (2018), "The Ins and Outs of Mathematical Explanation," The Mathematical Intelligencer 40(4), 26-29. https://doi.org/10.1007/s00283-018-9799-1

Diestel R. (2005), Graph Theory, New York: Springer.

Dieveney P. (2018), "Scientific Explanation, Unifying Mathematics, and Indispensability Arguments (A New Cicada MES),” Synthese, DOI: 10.1007/s11229-018-01979-9.

Drekalović V. (2016), "Two Weak Points of the Enhanced Indispensability Argument Domain of the Argument and Definition of Indispensability," Organon F 23(3), 280-298.

Drekalović V., Žarnić B. (2018), "Which Mathematical Objects are Referred to by the Enhanced Indispensability Argument?,” Journal for General Philosophy of Science 49(1), 121-126. https://doi.org/10.1007/s10838-017-9381-o

Drekalović V. (2018), "Is the Enhanced Indispensability Argument a Useful Tool in the Hands of Platonists?," Philosophia 47(4), 1111-1126. https://doi.org/10.1007/s11406-018-0033-3

Düntsch I., Gediga G. (2000), Sets, Relations, Functions, Bangor: Methoסos Publishers.

Goles E., Schulz O., Markus M. (2001), "Prime Number Selection of Cycles in a Predator-Prey Model," Complexity 6(4), 33-38. https://doi.org/10.1002/cplx.1040

Hales T. C. (2001), "The Honeycomb Conjecture," Discrete and Computational Geometry, 25(1), 1-22.

Lange M. (2013), "What Makes a Scientific Explanation Distinctively Mathematical?," British Journal for the Philosophy of Science 64(3), 485-511. https://doi.org/10.1093/ bjps/axso12 
Liggins D. (2008), "Quine, Putnam, and the 'Quine-Putnam' Indispensability Argument," Erkenntnis 68(1), 113-127. https://doi.org/10.1007/s10670-007-9081-y

Lloyd M., Dybas H. (1966), “The Periodical Cicada Problem I. Population Ecology,” Evolution 20(2), 133-149. https://doi.org/10.1111/j.1558-5646.1966.tbo3350.x

Maddy P. (1990), Realism in Mathematics, New York: Oxford University Press. https:// doi.org/10.1093/019824035X.001.0001

Panza M., Sereni A. (2013), Plato's Problem: An Introduction to Mathematical Platonism, New York: Palgrave Macmillan. https://doi.org/10.1057/9781137298133

Pincock C. (2012), Mathematics and Scientific Representation, Oxford: Oxford University Press. https://doi.org/10.1093/acprof:oso/9780199757107.001.0001

Smullyan R. (1992), Gödel's Incompleteness Theorems, Oxford: Oxford University Press.

Tallant J. (2013), “Optimus Prime: Paraphrasing Prime Number Talk," Synthese 190(12), 2065-2083. https://doi.org/10.1007/s11229-011-9959-8

Varro M. T. (1934), On Agriculture, trans. by W. D. Hooper, Harrison Boyd Ash, Cambridge, MA: Harvard University Press (Loeb Classical Library). 